\title{
Reaching the Ideal Glass in Polymer Spheres: Thermodynamics and Vibrational Density of States
}

\author{
Xavier Monnier, ${ }^{1}$ Juan Colmenero $\odot,{ }^{1,2,3}$ Marcel Wolf $\odot,{ }^{4}$ and Daniele Cangialosi ${ }^{1,3, *}$ \\ ${ }^{1}$ Donostia International Physics Center, Paseo Manuel de Lardizabal 4, 20018 San Sebastián, Spain \\ ${ }^{2}$ Departamento de Fisica de Materiales (UPV/EHU), Apartado 1072, 20080 San Sebastián, Spain \\ ${ }^{3}$ Centro de Fisica de Materiales (CSIC-UPV/EHU) Paseo Manuel de Lardizabal 5, 20018 San Sebastián, Spain \\ ${ }^{4}$ Heinz Maier-Leibnitz Zentrum (MLZ), Lichtenbergstrasse 1 85747, Garching, Germany
}

(Received 8 September 2020; revised 4 December 2020; accepted 17 February 2021; published 19 March 2021)

\begin{abstract}
The existence of an ideal glass and the resolution to the Kauzmann paradox is a long-standing open question in materials science. To address this problem, we exploit the ability of glasses with large interfacial area to access low energy states. We submit aggregates of spheres of a polymeric glass former to aging well below their glass transition temperature, $T_{g}$; and characterize their thermodynamic state by calorimetry, and the vibrational density of state (VDOS) by inelastic neutron scattering (INS). We show that, when aged at appropriate temperatures, glassy spheres attain a thermodynamic state corresponding to an ideal glass in time scales of about one day. In this state, the boson peak, underlying the deviation from the Debye level of the VDOS, is essentially suppressed. Our results are discussed in the framework of the link between the macroscopic thermodynamic state of glasses and their vibrational properties.
\end{abstract}

DOI: 10.1103/PhysRevLett.126.118004

A liquid can be cooled down below its melting temperature, provided that large cooling rates are used to avoid crystallization. Conventional supercooled liquids generally exhibit a thermodynamic state independent of the thermal history [1]. Further cooling leads to the formation of a glass, whose thermodynamic state profoundly depends on the way this is formed and on its thermal history [2]. The temperature at which the supercooled liquid loses equilibrium - the so-called glass transition temperature, $T_{g}$-which defines the potential energy of the glass (see Fig. 1), progressively decreases with the cooling rate. Once in the glassy state, spontaneous evolution towards low energy states takes place. Accessing these states in glasses may convey insights of utmost importance to unveil the nature of the glass transition.

Activity in this sense was stimulated long ago by the analysis of Kauzmann on several glass formers [3]. He noticed that, due to its large specific heat, the entropy of a supercooled liquid decreases on cooling faster than that of the corresponding crystal. Hence, there would be a temperature, addressed as the Kauzmann temperature, $T_{K}$, at which the supercooled liquid and the crystal entropies match. The existence of a supercooled liquid below $T_{K}$ would imply an entropy catastrophe with the unphysical scenario of a liquid having smaller entropy than that of the corresponding crystal. To avoid this paradoxical scenario, it has been proposed [4-6] and largely debated [7-14], that a true second order thermodynamic transition, the so-called "ideal" glass transition, at $T_{K}$ would take place. The existence of such a transition would mean the emergence of an ideal glass residing at the bottom of Goldstein's free energy landscape [15], excluding the crystal occupying the ultimate minimum (see Fig. 1). The thermodynamic state of a glass can be defined via the so-called fictive temperature, $T_{f}$ [16], the temperature of the intercept of the extrapolated

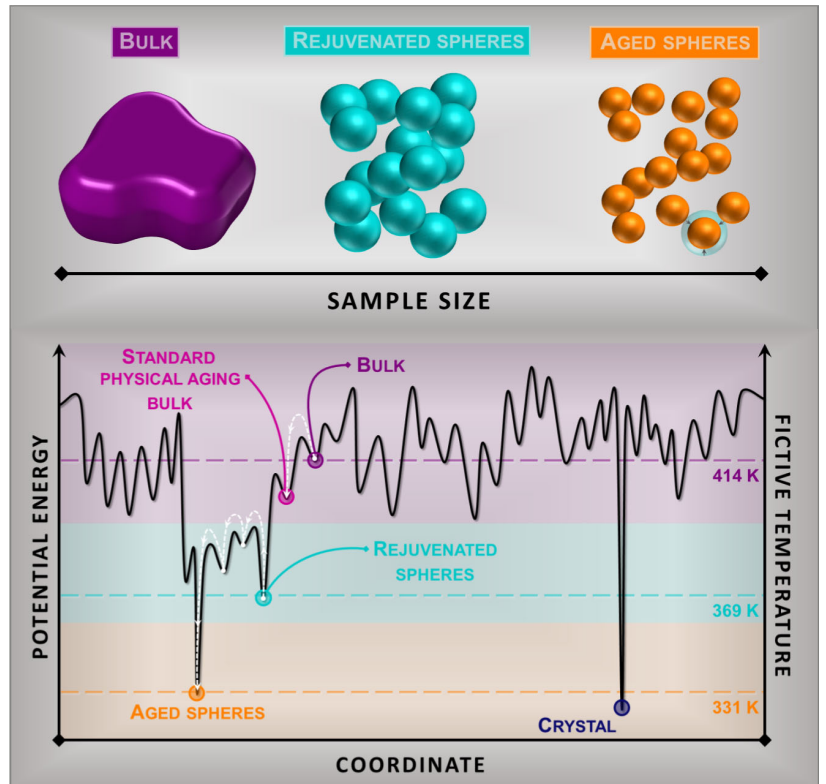

FIG. 1. Schematic representation of the potential energy landscape and the corresponding $T_{f}$, for bulk and spheres glasses. Bulk, rejuvenated, and aged spheres are located at high, intermediate, and low energetic minima in the landscape, respectively. The scheme shows how aging bulk glasses only allows limited energy decrease, whereas massive decrease takes place upon aging spheres. 
glass and supercooled equilibrium lines. $T_{f} \approx T_{K}$ defines the thermodynamic state of an "ideal glass," while $T_{f} \approx T_{g}$ that for a "fresh glass," obtained by cooling at a certain rate.

Attaining information on the ideal glass transition is hampered by the transformation of the supercooled liquid into a glass. Hence, reducing the energy requires the spontaneous evolution of the glass towards the equilibrium supercooled state, a phenomenon known as physical aging [17-19]. However, accessing low energies in bulk glasses by physical aging generally involves unfeasibly large time scales. For instance, glassy amber aged for millions of years [20,21] exhibits $T_{f}$ significantly lower than $T_{g}$ but still well above $T_{K}$. Aging bulk glasses over laboratory time scales only allows limited reduction of the glass energy (see Fig. 1).

Recently other routes have been explored to access low energy states $[22,23]$. Among them, a recently developed one relies on the ability of glasses with large free interface [24] to reach equilibrium in time scales way shorter than bulk glasses [25,26]. This is for instance evidenced by the finding of accelerated aging found in glasses of different nature having in common the large amount of free interface $[27,28]$. The efficiency of these systems to approach or maintain equilibrium has been explained accounting for models based on free volume holes diffusion [29,30]; whose evidence was directly verified in colloidal glasses [31]. In this way, different polymeric glasses have been shown to exhibit $T_{f}$ reductions as large as several tens of Kelvin [32] and in one specific case, that is, $30 \mathrm{~nm}$ thick polystyrene films as low as $T_{f} \approx T_{K}$ [12], thus creating glasses that by standard aging would take geological time scales to attain these low $T_{f}$.

Apart from the thermodynamic view, the achievement of low energy glasses has generated intense debate on how the vibrational density of state (VDOS) of a glass depends on its thermodynamic state [33]. The typical signature of the VDOS in disordered systems, including glasses, is the presence of the boson peak, that is, a low frequency excess of the VDOS with respect to the Debye level, generally found in crystals [34]. The boson peak has been interpreted either as arising from (quasi)localized modes [35] or being associated with random fluctuations of force constant originating from the intrinsic disordered nature of glasses [36]. Several studies on low $T_{f}$ glasses, including amber aged during geological time scales [21,37] and vapor deposited glasses [38,39], show significant depletion of the boson peak, which is explained by the decrease in the disorder of the glass. Hence, a question of utmost importance is whether the absence of disorder originating from the vanishing configurational entropy - the entropy depleted of its vibrational component - of the ideal glass with $T_{f}=T_{K}$ would result in the complete fading of the boson peak, in analogy to crystals with zero configurational entropy.

In this work, we exploit the ability of glasses with a large amount of free interface to access low energy states and characterize their VDOS by inelastic neutron scattering (INS) (see Supplemental Material [40], which includes Refs. [41-44]). We magnify the free interfacial area by employing aggregates of spheres made of a polymeric glass former, poly( 4 -tert-butyl styrene) (PtBS) with diameter $\approx 150 \mathrm{~nm}$. This is a model system of glass former chosen for its ability to form morphologically stable spheres. However, it is worth to remark that the approach based on enhancing the free interfacial area may be in principle extended to a diverse range of glass formers, provided that equilibrium bulk behavior is maintained. This is true in those cases where the typical length scale of the sample is large enough that the equilibrium thermodynamics is essentially bulklike. Spheres with diameter $150 \mathrm{~nm}$ fulfil this criterion as extensively discussed in the Supplemental Material [40], which includes Refs. [45-58]. Characterizing the time evolution of the enthalpy, that is, the energy at constant pressure, in terms of $T_{f}$ during physical aging by means of differential scanning calorimetry (DSC), we find that accessing low energy states well below $T_{g}$ is triggered by a fast mechanism of equilibrium recovery, widely characterized in recent years $[12,59,60]$. This allows massive decrease of $T_{f}$ down to the ideal glass. In this state, we find that the boson peak is essentially suppressed.

We selected $\mathrm{P} t \mathrm{BS}$ as a model material because its vitrification kinetics and molecular mobility were previously deeply characterized $[32,61,62]$. Furthermore, $\mathrm{P} t \mathrm{BS}$, as a result of its lack of tacticity, does not crystallize under any condition as discussed in detail in the Supplemental Material [40], which includes Ref. [63]. PtBS spheres were obtained by flash precipitation [64]. Their enthalpic state and VDOS were characterized by DSC and INS. Experimental details on the production of spheres and their AFM characterization, and on DSC and INS are presented in Supplemental Material [40].

The evolution of the PtBS thermodynamic state during aging in the glassy state is presented in Fig. 2, which shows specific heat scans for the spheres at different aging times at $253 \mathrm{~K}$ as a showcase. A similar behavior was found for the other aging temperatures [40]. Physical aging results in the development of a pronounced endothermic overshoot, increasing in magnitude with aging time, whose onset is located $\approx 100 \mathrm{~K}$ below $T_{g}$ and with breadth encompassing a wide range of temperatures up to $T_{g}$. This feature is well documented in glasses of different nature aged substantially below $T_{g}[12,60,65-71]$, and is explained taking into account a fast mechanism of equilibrium recovery with relatively low activation energy and a time scale much smaller than that of the main $\alpha$ relaxation $[59,72]$. These results point toward the universality of a fast evolution to a relative minimum in the free energy, theoretically identified with the concept of marginal glass [73]. Other theoretical approaches also based on general features of the heterogeneity of glasses [74,75] or polymer specific 


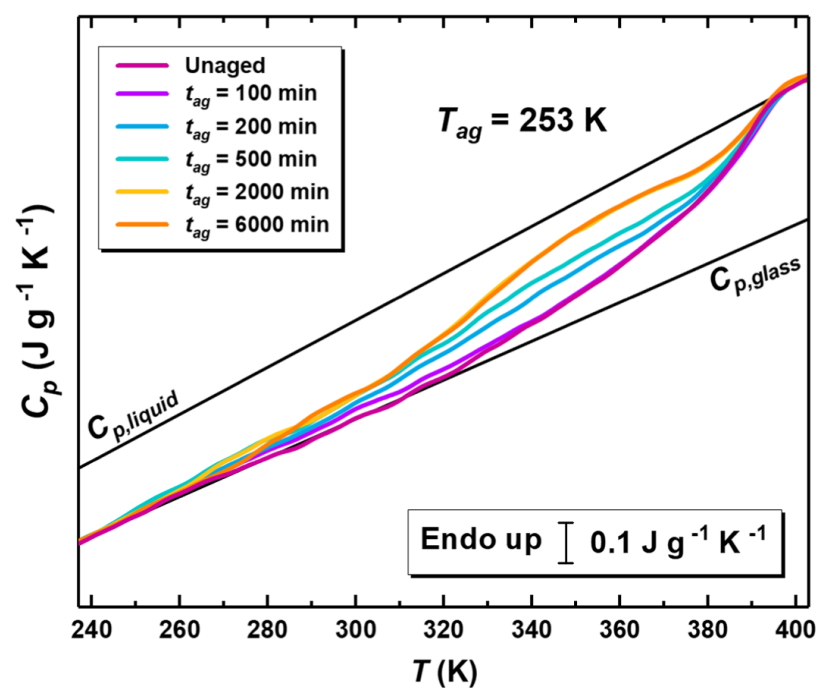

FIG. 2. Specific heat versus temperature measured by DSC upon heating at $10 \mathrm{~K} \mathrm{~min}^{-1}$ for spheres of $\mathrm{P} t \mathrm{BS}$ aged at $253 \mathrm{~K}$ for different times.

approaches [76] also allow describing the nature of the fast mechanism of equilibrium recovery.

Specific heat scans on samples aged at different temperatures and aging times allow us to determine the aging time evolution of the glass enthalpic state in terms of $T_{f}$, as shown in Fig. 3. The first important feature of this plot is that, at the beginning of the aging process after cooling at $10 \mathrm{~K} / \mathrm{min}, \mathrm{P} t \mathrm{BS}$ spheres exhibit drastic reduction in $T_{f}$ $\left(\approx T_{g}\right)$ with respect to bulk $\mathrm{P} t \mathrm{BS}$. Importantly such

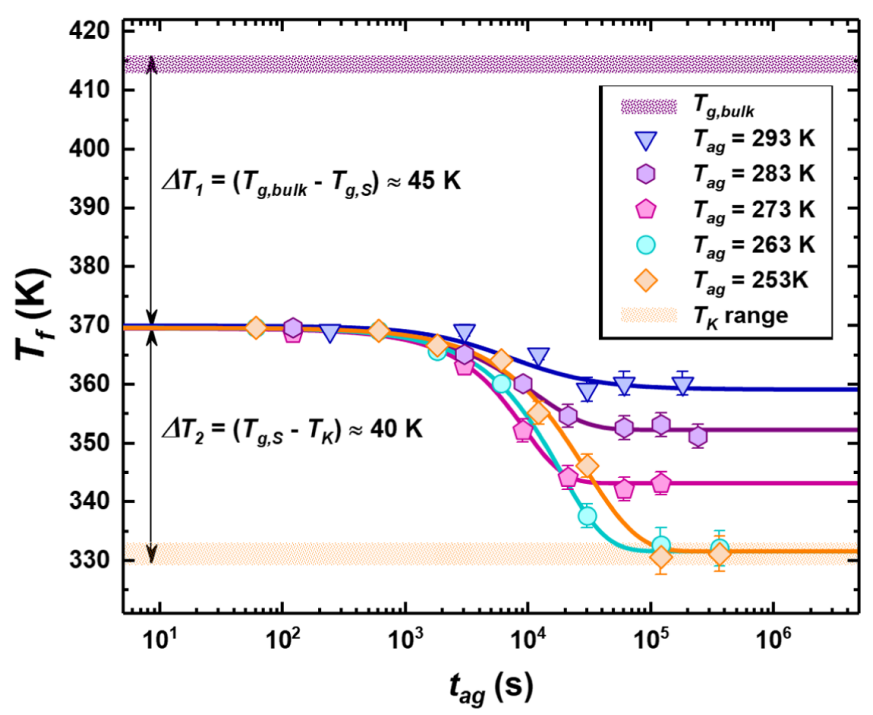

FIG. 3. Evolution of $T_{f}$ as a function of aging time at the indicated aging temperatures. The upper purple hatched area indicates bulk $\mathrm{P} t \mathrm{BS} T_{g}$ and the lower orange hatched area the temperature range below which no further $T_{f}$ decrease is allowed, which is therefore identified with $T_{K}$. The solid lines are guides for the eye to highlight the long term aging plateau reached at each aging temperature. reduction does not originate from a change in the equilibrium molecular mobility that exhibits bulk behavior (see Supplemental Material [40], which includes Ref. [77]), in line with previous results on polymer spheres $[32,78]$. This result indicates that vitrification on cooling is delayed to lower temperatures in spheres thanks to their ability to maintain equilibrium more efficiently than the bulk. This ability is also highlighted by the massive $T_{f}$ decrease to a plateau in time scales of days. Lowering the aging temperature from 293 to $263 \mathrm{~K}$ results in a systematic decrease of the plateau value of $T_{f}$. In contrast, once the aging temperature is decreased below $263 \mathrm{~K}$, the $T_{f}$ at the long aging time plateau remains constant.

Importantly, the $T_{f}$ attained upon aging at $T \leq 263 \mathrm{~K}$ conditions is $\approx 330 \mathrm{~K}$, which is largely below that of bulk unaged $\mathrm{P} t \mathrm{BS} \quad\left(T_{f} \approx 415 \mathrm{~K}\right)$. This reduction would be unattainable by aging in conventional bulk glasses on experimentally accessible time scales. Furthermore, though the $T_{K}$ of $\mathrm{P} t \mathrm{BS}$ is unknown, the invariance of $T_{f}$ at the plateau after aging at $T \leq 263 \mathrm{~K}$ implies that no further decrease is allowed and, therefore, this $T_{f}$ corresponds to $T_{K}$. Even if an indirect determination $T_{K}$ of $\mathrm{P} t \mathrm{BS}$ is missing, it is worth remarking that the distance from $T_{g}$ is about $85 \mathrm{~K}$, which is of the same order of that of polystyrene (PS), about $90 \mathrm{~K}$ [12], for which the $T_{K}$ is well defined [79]. Hence the ideal glass is reached after mild aging at $T \leq 263 \mathrm{~K}$, as clearly evidenced in the energetic plot presented in Fig. 4 in terms of the excess enthalpy with respect to the lowest enthalpy state with $T_{f}=330 \mathrm{~K}$ (left axis), or equivalently the fictive temperature (right axis).

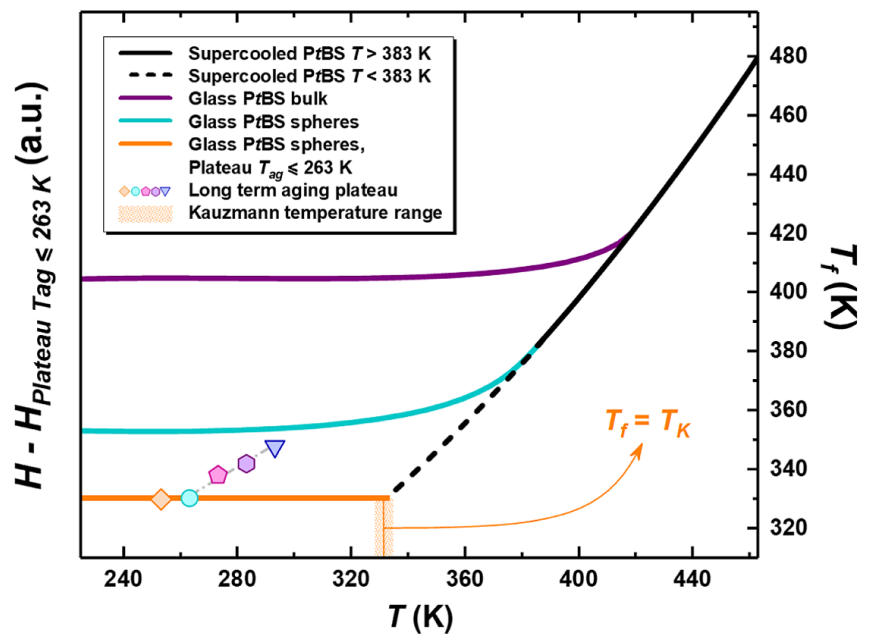

FIG. 4. Enthalpy in excess to that corresponding to $T_{f}=$ $330 \mathrm{~K}$ (left axis) and fictive temperature (right axis) as a function of temperature for bulk $\mathrm{P} t \mathrm{BS}$ and $\mathrm{P} t \mathrm{BS}$ spheres in different conditions. Points are for the long term aging plateau of $\mathrm{P} t \mathrm{BS}$ spheres. Lines are obtained from the temperature dependence of the specific heat on cooling at $10 \mathrm{~K} /$ min bulk PtBS (violet) and spheres (cyan); and both systems in the melt state (black line). The dashed black line is the extrapolation to low temperatures of the experimental melt line. 


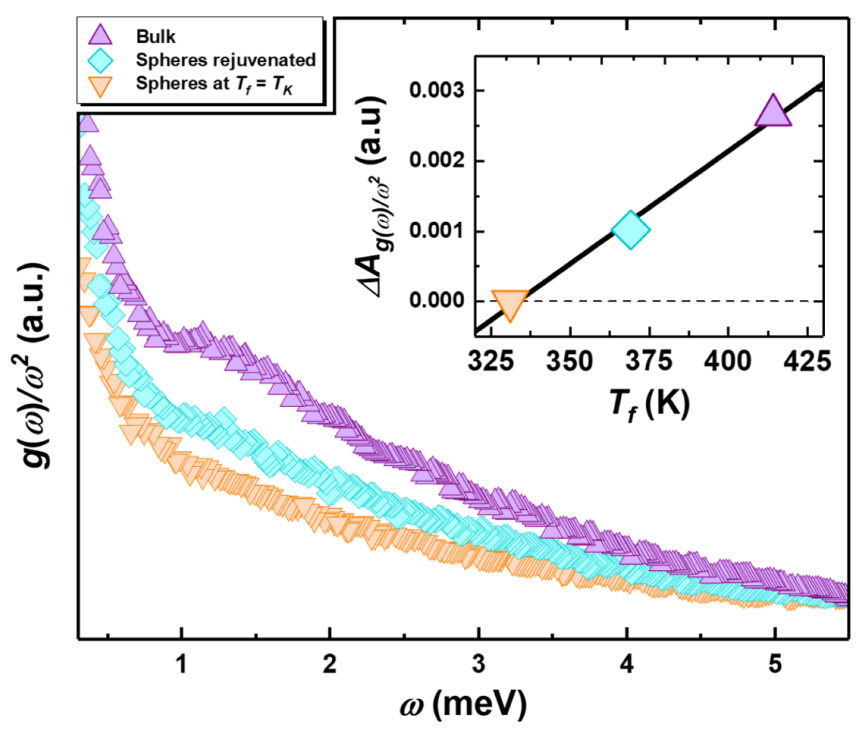

FIG. 5. Reduced VDOS for bulk PtBS, and spheres rejuvenated and aged to $T_{f} \approx T_{K}$. The inset shows the area underlying between each sample and that of $\mathrm{P} t \mathrm{BS}$ spheres with $T_{f} \approx T_{K}$ as a function of the fictive temperature.

This shows that $\mathrm{P} t \mathrm{BS}$ spheres aged to a plateau exhibit a kink in the enthalpy and $T_{f}$. This can be interpreted as underlying the transformation from a standard to an ideal glass and is in line with the assertion that $T_{f} \approx T_{K}=330 \mathrm{~K}$.

The macroscopic thermodynamic overview on $\mathrm{P} t \mathrm{BS}$ spheres aged deep in the glassy state is complemented by insights of extraordinary importance on the VDOS obtained by INS. Figure 5 represents the reduced VDOS, $g(\omega) / \omega^{2}$ - excluding the elastic peak mainly visible at low frequencies-obtained from the intermediate scattering function, $S(q, \omega)$, measured at $50 \mathrm{~K}$ [40]. The latter is directly measured by INS. Bulk PtBS measured immediately after cooling with $10 \mathrm{~K} /$ min shows the typical excess VDOS evidenced by the boson peak anomaly at $1 \div 1.5 \mathrm{meV}$. This is significantly depleted in rejuvenated PtBS spheres, that is, taken above $T_{g}$ and immediately cooled to $50 \mathrm{~K}$. The boson peak is essentially suppressed after aging the spheres at $253 \mathrm{~K}$ for $t_{a g} \approx 10^{5} \mathrm{~s}$, which is sufficient to achieve the lowest $T_{f}=330 \mathrm{~K}$, identified with $T_{K}$. The inset of Fig. 5 establishes the connection between the VDOS and the thermodynamic state of the glass. It illustrates that the variation of the area below the reduced VDOS of both bulk $\mathrm{P} t \mathrm{BS}$ and rejuvenated spheres in excess with respect to that of spheres aged to $T_{f}=330 \mathrm{~K}$ scales in a linear fashion with the glass thermodynamic state in terms of $T_{f}$.

While other studies showed that thermodynamic states corresponding to the ideal glass can be attained [12], the suppression of the boson peak observed experimentally in the ideal glass is unprecedented. This result is in line with the finding that the magnitude of the boson peak scales with the degree of disorder in the glass, i.e., the configurational entropy, which is directly connected to $T_{f}$ [33]. Hence, the boson peak progressively decreases from hyperquenched glasses [80] to hyperaged [21,37], vapor deposited [38,39] and pressure densified [81] glasses, passing from weakly aged systems $[33,82,83]$ and eventually disappears in the ideal glass of the present study. The connection between VDOS and $T_{f}$ shown in the inset of Fig. 5 proves that the microscopic vibrational properties are intimately linked to the macroscopic thermodynamic state of the glass in terms of its degree of disorder, that is, its configurational entropy [33].

The presence of large free interface is a key parameter to obtain the ideal glass, exhibiting suppression of the boson peak. Hence, our results can be discussed in light of the activity pursued on the characterization of the VDOS of glasses subjected to geometrical confinement [84]. Specifically, it is worth mentioning those works on polymer glasses subjected to "soft confinement," where large free interface is present. Studies by inelastic neutron scattering on molecular glass former propylene glycol nanodroplets with diameter as low as $\sim 4 \mathrm{~nm}$ suspended in decalin show disappearance of the boson peak [85]. A similar outcome was found in polymer thin films with a free interface, showing significant depression of the VDOS with respect to the bulk [86]. These studies, however, do not provide a characterization of the thermodynamic state attained in these kinds of confinement. We insinuate that the suppression of the boson peak and its relation to the corresponding energy state is a universal feature of all glasses exhibiting large amount of free interface. Hence, new impetus in the search of the ideal glass is expected in glasses of different nature, provided that their free interfacial area is magnified.

In conclusion, we have shown that increasing glasses' free interface induces a tremendous reduction of the time scale to recover equilibrium. In appropriate conditions via a relaxation process with finite time scale deep in the glassy state, this acceleration allows attaining glass energies relevant to detect a kink in the enthalpy, identified with the transformation from the standard into the ideal glass. Concomitantly, we have shown for the first time that the boson peak disappears from the low frequency VDOS in the ideal glassy state, thus proving what has been usually conjectured.

D. C. and J. C. acknowledge financial support from the Project No. PGC2018-094548-B-I00 (MICINN-Spain and FEDER-UE) and the Project No. IT-1175-19 (Basque Government).

*daniele.cangialosi@ehu.eus

[1] J. W. P. Schmelzer and I. S. Gutzow, Glasses and the Glass Transition (Wiley-VCH, Weinheim, 2011).

[2] D. Cangialosi, Dynamics and thermodynamics of polymer glasses, J. Phys. Condens. Matter 26, 153101 (2014). 
[3] W. Kauzmann, The nature of the glassy state and the behavior of liquids at low temperatures, Chem. Rev. 43, 219 (1948).

[4] J. H. Gibbs and E. A. DiMarzio, Nature of the glass transition and the glassy state, J. Chem. Phys. 28, 373 (1958).

[5] V. Lubchenko and P. G. Wolynes, Theory of structural glasses and supercooled liquids, Annu. Rev. Phys. Chem. 58, 235 (2007).

[6] M. Mézard and G. Parisi, Thermodynamics of Glasses: A First Principles Computation, Phys. Rev. Lett. 82, 747 (1999).

[7] F. H. Stillinger, P. G. Debenedetti, and T. M. Truskett, The kauzmann paradox revisited, J. Phys. Chem. B 105, 11809 (2001).

[8] M. Ozawa, W. Kob, A. Ikeda, and K. Miyazaki, Equilibrium phase diagram of a randomly pinned glass-former, Proc. Natl. Acad. Sci. U.S.A. 112, 6914 (2015).

[9] M. Ozawa, A. Ikeda, K. Miyazaki, and W. Kob, Ideal Glass States are Not Purely Vibrational: Insight from Randomly Pinned Glasses, Phys. Rev. Lett. 121, 205501 (2018).

[10] L. Berthier, P. Charbonneau, D. Coslovich, A. Ninarello, M. Ozawa, and S. Yaida, Configurational entropy measurements in extremely supercooled liquids that break the glass ceiling, Proc. Natl. Acad. Sci. U.S.A. 114, 11356 (2017).

[11] C. P. Royall, F. Turci, S. Tatsumi, J. Russo, and J. Robinson, The race to the bottom: Approaching the ideal glass?, J. Phys. Condens. Matter 30, 363001 (2018).

[12] V. M. Boucher, D. Cangialosi, A. Alegria, and J. Colmenero, Reaching the ideal glass transition by aging polymer films, Phys. Chem. Chem. Phys. 19, 961 (2017).

[13] H. Yoon and G. B. McKenna, Testing the paradigm of an ideal glass transition: Dynamics of an ultrastable polymeric glass, Sci. Adv. 4, eaau5423 (2018).

[14] M. S. Beasley, C. Bishop, B. J. Kasting, and M. D. Ediger, Vapor-deposited ethylbenzene glasses approach ideal glass density, J. Phys. Chem. Lett. 10, 4069 (2019).

[15] M. Goldstein, Viscous liquids and the glass transition: A potential energy barrier picture, J. Chem. Phys. 51, 3728 (1969).

[16] C. T. Moynihan, P. B. Macedo, C. J. Montrose, P. K. Gupta, M. A. De Bolt, J. F. Dill, B. E. Dom, P. W. Drake, A. J. Eastel, P. B. Elterman, R. P. Moeller, H. Sasabe, and J. A. Wilder, Structural relaxation in vitreous materials, Ann. N.Y. Acad. Sci. 279, 15 (1976).

[17] L. C. E. Struik, Physical Aging in Amorphous Polymers and Other Materials (Technische Hogeschool, Delft., 1977).

[18] D. Cangialosi, V. M. Boucher, A. Alegria, and J. Colmenero, Physical aging in polymers and polymer nanocomposites: Recent results and open questions, Soft Matter 9, 8619 (2013).

[19] A. J. Kovacs, Glass transition in amorphous polymers: A phenomenological study, Fortsch. Hochpolym. Fo. 3, 394 (1963).

[20] J. Zhao, S. L. Simon, and G. B. McKenna, Using 20million-year-old amber to test the super-arrhenius behaviour of glass-forming systems, Nat. Commun. 4, 1783 (2013).

[21] T. Pérez-Castañeda, R. J. Jiménez-Riobóo, and M. A. Ramos, Two-Level Systems and Boson Peak Remain Stable in 110-Million-Year-Old Amber Glass, Phys. Rev. Lett. 112, 165901 (2014).
[22] S. F. Swallen, K. L. Kearns, M. K. Mapes, Y. S. Kim, R. J. McMahon, M. D. Ediger, T. Wu, L. Yu, and S. Satija, Organic glasses with exceptional thermodynamic and kinetic stability, Science 315, 353 (2007).

[23] J. Ràfols-Ribé, P.-A. Will, C. Hänisch, M. GonzalezSilveira, S. Lenk, J. Rodríguez-Viejo, and S. Reineke, High-performance organic light-emitting diodes comprising ultrastable glass layers, Sci. Adv. 4, eaar8332 (2018).

[24] S. Napolitano, C. Rotella, and M. Wubbenhorst, Can thickness and interfacial interactions univocally determine the behavior of polymers confined at the nanoscale?, ACS Macro Lett. 1, 1189 (2012).

[25] D. Cangialosi, A. Alegria, and J. Colmenero, Effect of nanostructure on the thermal glass transition and physical aging in polymer materials, Prog. Polym. Sci. 54-55, 128 (2016).

[26] M.-C. Ma and Y.-L. Guo, Physical properties of polymers under soft and hard nanoconfinement: A review, Chinese Journal of Polymer Science 38, 565 (2020).

[27] V. M. Boucher, D. Cangialosi, A. Alegria, J. Colmenero, I. Pastoriza-Santos, and L. M. Liz-Marzan, Physical aging of polystyrene/gold nanocomposites and its relation to the calorimetric tg depression, Soft Matter 7, 3607 (2011).

[28] A. Sepulveda, E. Leon-Gutierrez, M. Gonzalez-Silveira, C. Rodriguez-Tinoco, M. T. Clavaguera-Mora, and J. Rodriguez-Viejo, Accelerated Aging in Ultrathin Films of a Molecular Glass Former, Phys. Rev. Lett. 107, 025901 (2011).

[29] M. S. McCaig, D. R. Paul, and J. W. Barlow, Effect of film thickness on the changes in gas permeability of a glassy polyarylate due to physical agingpart II. Mathematical model, Polymer 41, 639 (2000).

[30] D. Cangialosi, V. M. Boucher, A. Alegria, and J. Colmenero, Free volume holes diffusion to describe physical aging in poly(mehtyl methacrylate)/silica nanocomposites, J. Chem. Phys. 135, 014901 (2011).

[31] X. Cao, H. Zhang, and Y. Han, Release of free-volume bubbles by cooperative-rearrangement regions during the deposition growth of a colloidal glass, Nat. Commun. 8, 362 (2017).

[32] X. Monnier and D. Cangialosi, Thermodynamic Ultrastability of a Polymer Glass Confined at the Micrometer Length Scale, Phys. Rev. Lett. 121, 137801 (2018).

[33] C. A. Angell, Y. Yue, L.-M. Wang, J. R. Copley, S. Borick, and S. Mossa, Potential energy, relaxation, vibrational dynamics and the boson peak, of hyperquenched glasses, J. Phys. Condens. Matter 15, S1051 (2003).

[34] H. Shintani and H. Tanaka, Universal link between the boson peak and transverse phonons in glass, Nat. Mater. 7, 870 (2008).

[35] B. B. Laird and H. R. Schober, Localized Low-Frequency Vibrational Modes in a Simple Model Glass, Phys. Rev. Lett. 66, 636 (1991).

[36] W. Schirmacher, G. Diezemann, and C. Ganter, Harmonic Vibrational Excitations in Disordered Solids and the "Boson Peak", Phys. Rev. Lett. 81, 136 (1998).

[37] E. Pogna, A. Chumakov, C. Ferrante, M. Ramos, and T. Scopigno, Tracking the connection between disorder and energy landscape in glasses using geologically hyperaged amber, J. Phys. Chem. Lett. 10, 427 (2019). 
[38] T. Pérez-Castañeda, C. Rodríguez-Tinoco, J. RodríguezViejo, and M. A. Ramos, Suppression of tunneling two-level systems in ultrastable glasses of indomethacin, Proc. Natl. Acad. Sci. U.S.A. 111, 11275 (2014).

[39] S. Singh, M. D. Ediger, and J. J. De Pablo, Ultrastable glasses from in silico vapour deposition, Nat. Mater. 12, 139 (2013).

[40] See Supplemental Material at http://link.aps.org/ supplemental/10.1103/PhysRevLett.126.118004 for the description of the production of spheres and the AFM characterization, details on calorimetric and neutron scattering experiments and a discussion based on numerous previous studies showing the effect of free interfaces on equilibrium thermodynamics.

[41] W. Lohstroh and Z. Evenson, Toftof: Cold neutron time-offlight spectrometer, J. Large-Scale Res. Facil. 1, A15 (2015).

[42] O. Arnold, J.-C. Bilheux, J. Borreguero, A. Buts, S. I. Campbell, L. Chapon, M. Doucet, N. Draper, R. F. Leal, M. Gigg et al., Mantiddata analysis and visualization package for neutron scattering and $\mu$ sr experiments, Nucl. Instrum. Methods Phys. Res., Sect. A 764, 156 (2014).

[43] R. Zorn, P. Szymoniak, M. A. Kolmangadi, M. Wolf, D. A. Alentiev, M. Bermeshev, M. Böhning, and A. Schönhals, Low frequency vibrational density of state of highly permeable super glassy polynorbornenes-the boson peak, Phys. Chem. Chem. Phys. 22, 18381 (2020).

[44] J. Colmenero, A. J. Moreno, and A. Alegría, Neutron scattering investigations on methyl group dynamics in polymers, Prog. Polym. Sci. 30, 1147 (2005).

[45] E. Kang, H. Kim, L. A. Gray, D. Christie, U. Jonas, B. Graczykowski, E. M. Furst, R. D. Priestley, and G. Fytas, Ultrathin shell layers dramatically influence polymer nanoparticle surface mobility, Macromolecules 51, 8522 (2018).

[46] E. Kang, B. Graczykowski, U. Jonas, D. Christie, L. A. Gray, D. Cangialosi, R. D. Priestley, and G. Fytas, Shell architecture strongly influences the glass transition, surface mobility, and elasticity of polymer core-shell nanoparticles, Macromolecules 52, 5399 (2019).

[47] R. P. White and J. E. G. Lipson, Thermodynamic treatment of polymer thin-film glasses, Phys. Rev. E 84, 041801 (2011).

[48] R. P. White, C. C. Price, and J. E. G. Lipson, Effect of interfaces on the glass transition of supported and freestanding polymer thin films, Macromolecules 48, 4132 (2015)

[49] W. Wallace, N. B. Tan, W. Wu, and S. Satija, Mass density of polystyrene thin films measured by twin neutron reflectivity, J. Chem. Phys. 108, 3798 (1998).

[50] J. Algers, R. Suzuki, T. Ohdaira, and F. H. Maurer, Characterization of free volume and density gradients of polystyrene surfaces by low-energy positron lifetime measurements, Polymer 45, 4533 (2004).

[51] A. Van der Lee, L. Hamon, Y. Holl, and Y. Grohens, Density profiles in thin pmma supported films investigated by $\mathrm{x}$-ray reflectometry, Langmuir 17, 7664 (2001).

[52] R. Inoue, K. Kawashima, K. Matsui, T. Kanaya, K. Nishida, G. Matsuba, and M. Hino, Distributions of glass-transition temperature and thermal expansivity in multilayered polystyrene thin films studied by neutron reflectivity, Phys. Rev. E 83, 021801 (2011).

[53] J. E. Pye and C. B. Roth, Above, below, and in-between the two glass transitions of ultrathin free-standing polystyrene films: Thermal expansion coefficient and physical aging, J. Polym. Sci. B 53, 64 (2015).

[54] Y. Shin, H. Lee, W. Lee, and D. Y. Ryu, Glass transition and thermal expansion behavior of polystyrene films supported on polystyrene-grafted substrates, Macromolecules 49, 5291 (2016).

[55] S. Napolitano, A. Pilleri, P. Rolla, and M. Wubbenhorst, Unusual deviations from bulk behavior in ultrathin films of poly (tert-butylstyrene): Can dead layers induce a reduction of $\mathrm{t} g$ ?, ACS Nano 4, 841 (2010).

[56] S. Peter, H. Meyer, and J. Baschnagel, Thickness-dependent reduction of the glass-transition temperature in thin polymer films with a free surface, J. Polym. Sci. B 44, 2951 (2006).

[57] P. Z. Hanakata, J. F. Douglas, and F. W. Starr, Local variation of fragility and glass transition temperature of ultra-thin supported polymer films, J. Chem. Phys. 137, 244901 (2012).

[58] J. Sarabadani, A. Milchev, and T. A. Vilgis, Structure and dynamics of polymer melt confined between two solid surfaces: A molecular dynamics study, J. Chem. Phys. 141, 044907 (2014).

[59] D. Cangialosi, V. M. Boucher, A. Alegría, and J. Colmenero, Direct Evidence of Two Equilibration Mechanisms in Glassy Polymers, Phys. Rev. Lett. 111, 095701 (2013).

[60] N. G. Perez-De Eulate and D. Cangialosi, The very longterm physical aging of glassy polymers, Phys. Chem. Chem. Phys. 20, 12356 (2018).

[61] C. J. Ellison, M. K. Mundra, and J. M. Torkelson, Impacts of polystyrene molecular weight and modification to the repeat unit structure on the glass transition- nanoconfinement effect and the cooperativity length scale, Macromolecules 38, 1767 (2005).

[62] N. G. Perez-de Eulate, M. Sferrazza, D. Cangialosi, and S. Napolitano, Irreversible adsorption erases the free surface effect on the tg of supported films of poly(4-tert-butylstyrene), ACS Macro Lett. 6, 354 (2017).

[63] K. Paeng and M. Ediger, Molecular motion in freestanding thin films of poly (methyl methacrylate), poly (4-tert-butylstyrene), poly ( $\alpha$-methylstyrene), and poly (2-vinylpyridine), Macromolecules 44, 7034 (2011).

[64] C. Zhang, V. J. Pansare, R. K. Prud'Homme, and R. D. Priestley, Flash nanoprecipitation of polystyrene nanoparticles, Soft Matter 8, 86 (2012).

[65] A. R. Berens and I. M. Hodge, Effects of annealing and prior history on enthalpy relaxation in glassy polymers. 1. Experimental study on poly(vinyl chloride), Macromolecules 15, 756 (1982).

[66] J. Fan, E. I. Cooper, and C. A. Angell, Glasses with strong calorimetric $\beta$-glass transitions and the relation to the protein glass transition problem, J. Phys. Chem. 98, 9345 (1994).

[67] D. P. B. Aji and G. P. Johari, Kinetic-freezing and unfreezing of local-region fluctuations in a glass structure observed by heat capacity hysteresis, J. Chem. Phys. 142, 214501 (2015). 
[68] L. Song, W. Xu, J. Huo, J.-Q. Wang, X. Wang, and R. Li, Two-step relaxations in metallic glasses during isothermal annealing, Intermetallics 93, 101 (2018).

[69] R. Androsch, K. Jariyavidyanont, and C. Schick, Enthalpy relaxation of polyamide 11 of different morphology far below the glass transition temperature, Entropy 21, 984 (2019).

[70] Q. Yang, S.-X. Peng, Z. Wang, and H.-B. Yu, Shadow glass transition as a thermodynamics signature of $\beta$ relaxation in hyper-quenched metallic glasses, Natl. Sci. Rev. 7, 1896 (2020).

[71] M. Gao and J. H. Perepezko, Separating $\beta$ relaxation from $\alpha$ relaxation in fragile metallic glasses based on ultrafast flash differential scanning calorimetry, Phys. Rev. Mater. 4, 025602 (2020).

[72] V. M. Boucher, D. Cangialosi, A. Alegria, and J. Colmenero, Complex nonequilibrium dynamics of stacked polystyrene films deep in the glassy state, J. Chem. Phys. 146, 203312 (2017).

[73] P. Charbonneau, J. Kurchan, G. Parisi, P. Urbani, and F. Zamponi, Fractal free energy landscapes in structural glasses, Nat. Commun. 5, 3725 (2014).

[74] A. Wisitsorasak and P. G. Wolynes, Dynamical heterogeneity of the glassy state, J. Phys. Chem. B 118, 7835 (2014).

[75] E. Bouchbinder and J. S. Langer, Shear-transformation-zone theory of linear glassy dynamics, Phys. Rev. E 83, 061503 (2011).

[76] K. Ngai, S. Capaccioli, and L.-M. Wang, Segmental $\alpha$-relaxation for the first step and sub-rouse modes for the second step in enthalpy recovery in the glassy state of polystyrene, Macromolecules 52, 1440 (2019).

[77] S. Chandran, J. Baschnagel, D. Cangialosi, K. Fukao, E. Glynos, L. M. Janssen, M. Mueller, M. Muthukumar, U. Steiner, J. Xu, S. Napolitano, and G. Reiter, Processing pathways decide polymer properties at the molecular level, Macromolecules 52, 7146 (2019).

[78] N. G. Perez-de Eulate, V. Di Lisio, and D. Cangialosi, Glass transition and molecular dynamics in polystyrene nanospheres by fast scanning calorimetry, ACS Macro Lett. 6, 859 (2017).

[79] A. A. Miller, Excess thermodynamic quantities in linear polymer liquids, Macromolecules 3, 674 (1970).

[80] A. Monaco, A. I. Chumakov, Y.-Z. Yue, G. Monaco, L. Comez, D. Fioretto, W. Crichton, and R. Rüffer, Density of Vibrational States of a Hyperquenched Glass, Phys. Rev. Lett. 96, 205502 (2006).

[81] K. Niss, B. Begen, B. Frick, J. Ollivier, A. Beraud, A. Sokolov, V. N. Novikov, and C. Alba-Simionesco, Influence of Pressure on the Boson Peak: Stronger than Elastic Medium Transformation, Phys. Rev. Lett. 99, 055502 (2007).

[82] E. Duval, L. Saviot, L. David, S. Etienne, and J. Jal, Effect of physical aging on the low-frequency vibrational density of states of a glassy polymer, Europhys. Lett. 63, 778 (2003).

[83] R. Zorn, H. Yin, W. Lohstroh, W. Harrison, P. M. Budd, B. R. Pauw, M. Böhning, and A. Schönhals, Anomalies in the low frequency vibrational density of states for a polymer with intrinsic microporosity-the boson peak of pim-1, Phys. Chem. Chem. Phys. 20, 1355 (2018).

[84] R. Zorn, Boson peak in confined disordered systems, Phys. Rev. B 81, 054208 (2010).

[85] R. Zorn, M. Mayorova, D. Richter, and B. Frick, Inelastic neutron scattering study of a glass-forming liquid in soft confinement, Soft Matter 4, 522 (2008).

[86] R. Inoue, T. Kanaya, K. Nishida, I. Tsukushi, and K. Shibata, Inelastic Neutron Scattering Study of Low Energy Excitations in Polymer Thin Films, Phys. Rev. Lett. 95, 056102 (2005). 